\title{
Scalar field of non-paraxial Gaussian beams
}

\author{
Z. Ulanowski and I. K. Ludlow
}

Department of Physical Sciences, University of Hertfordshire, Hatfield, Herts AL10 9AB, UK.

\begin{abstract}
A family of closed form expressions for the scalar field of strongly focused Gaussian beams in oblate spheroidal coordinates is given. The solutions satisfy the wave equation and are free from singularities. The lowest order solution in the far field closely matches the energy density produced by a sine condition, high-aperture lens illuminated by a paraxial Gaussian beam. At the large waist limit it reduces to the paraxial Gaussian beam form. It is equivalent to the spherical wave of combined complex point source and sink but has the advantage of more direct interpretation.
\end{abstract}

OCIS codes: $140.3430,260.2110$.

There is much interest in obtaining descriptions of Gaussian beams extending beyond the paraxial case, prompted by many diverse uses of strongly focused laser beams in fields such as microscopy, optical information storage or optical trapping. A variety of infinite series, integral and approximate solutions have been proposed. However, as the beam divergence angle grows these models become either computationally onerous or increasingly inaccurate. A particularly simple description of Gaussian-like focused beams is possible in oblate spheroidal coordinates. ${ }^{1}$ The scalar field of the lowest order mode can be expressed as:

$$
\psi_{00}^{(1)}=\frac{\exp [k d(\eta+i \xi)]}{\exp (k d) k d(\eta+i \xi)},
$$

where $k=2 \pi / \lambda$ and $\xi, \eta$ and $\varphi$ are oblate spheroidal coordinates described in terms of Cartesian $x, y$ and $z$ as: ${ }^{2}$ 


$$
\begin{aligned}
& x=d \sqrt{1+\xi^{2}} \sqrt{1-\eta^{2}} \cos \varphi, \\
& y=d \sqrt{1+\xi^{2}} \sqrt{1-\eta^{2}} \sin \varphi, \\
& z=d \xi \eta .
\end{aligned}
$$

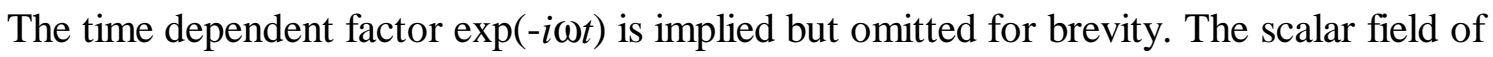
expression (1) is a solution of the wave equation and has many properties expected from a fundamental mode Gaussian beam beyond the paraxial approximation. ${ }^{1}$ However, it's major shortcomings are a circular singularity of radius $d$ in the beam waist plane and a discontinuity occurring on the "focal disc" circumscribed by the singularity, apparently violating energy conservation - see Fig. 1.

We observe that removal of both the singularity and the discontinuity can be accomplished by adding a second wave to give

$$
\psi_{00}=\psi_{00}^{(1)}-\frac{\exp [-k d(\eta+i \xi)]}{\exp (k d) k d(\eta+i \xi)}=2 \frac{\sin [k d(\xi-i \eta)]}{\exp (k d) k d(\xi-i \eta)} .
$$

The scalar field (3) is also an exact solution of the Helmholtz equation. It can be described as consisting of a superposition of the outgoing wave (1) and an incoming wave represented by the second term in (3). A similar interpretation in the context of a beam wave superposition in Cartesian coordinates has recently been given by Sheppard and Saghafi, ${ }^{3}$ who propose cancelling out the singularity due to a complex point source at $z=i z_{0}$ by the addition of a second, incoming wave associated with a sink point at the same location.

By substituting the coordinates (2) into the distance from the complex point source $R=\left[x^{2}+y^{2}+\left(z-i z_{0}\right)^{2}\right]^{1 / 2}$ and setting $z_{0}=d$ we obtain, after some algebra, the identity $R=d(\xi-i \eta)$. Apart from a constant factor, the spheroidal waves (1) and (3) can thus be shown to be identically equivalent to Deschamp's complex point source wave ${ }^{4}$ and the combined source and sink wave, ${ }^{3}$ respectively. This equivalence permits more intuitive interpretation of the complex point waves than is possible through the usual description as spherical waves associated with sources (or sinks) in complex space. The outgoing wavefronts can be naturally depicted as oblate ellipsoids of constant $\xi$ emanating from 
the focal disc predominantly in the direction of positive $z$ (Fig. 1). The incoming wavefronts similarly converge on, and sink into, the focal disc from the direction of negative $z$ - the waves exactly mirror each other upon time reversal. Together, the two waves form what is a physically realisable beam, continuous across the $z=0$ plane (Fig. 2). Unusual feature of the combined solution (3) is a standing wave component near the $z=0$ plane. This component grows in significance as $k d$ is reduced and represents interference of the "wings" of the incoming and outgoing waves. This is easily demonstrated be setting $\eta=0$ in expression (3), which corresponds to the $z=0$ plane outside the focal disc. We then obtain a pure standing wave characterised by the same wavelength as the incoming or outgoing waves - see Fig. 3. It should be noted, however, that producing such interference would require a focusing element subtending a solid angle greater than $2 \pi$.

In the plane of the beam waist and at the limit of large waist radius, i.e. for $k d>>1$, expression (3) can be reduced to the familiar paraxial Gaussian beam form

$$
\exp \left[-k\left(x^{2}+y^{2}\right) / 2 d\right] / k d
$$

by applying the binomial expansion for $\eta$ and neglecting terms of order greater than two. The parameter $d$ can now be identified with the Rayleigh range: $d=k w_{0}^{2} / 2$, where $w_{0}$ is the beam waist radius.

Inasmuch as the two terms in (3) are an incoming and an outgoing part of the same wave, they can be examined separately for the purpose of investigating boundary conditions. In the far field, as represented by $\xi \rightarrow \infty$, the incoming wave tends to:

$$
-\exp [-k d(1-\cos \alpha)] \exp (-i k r) / i k r
$$

since $\xi \rightarrow r / d$ and $\eta \rightarrow-\cos \alpha$, where $r$ and $\alpha$ are the distance from the origin and the angle made with the negative $z$ axis, respectively. As required, expression (5) represents a spherical wave with a centre at the origin and angular weighting $\exp [-k d(1-\cos \alpha)]$. The normalised irradiance of the incoming wave in the far field is then:

$$
\exp [-2 k d(1-\cos \alpha)]
$$


We can compare expression (6) to the irradiance on a reference sphere representing the principal surface of an aberration-free lens obeying the sine condition ${ }^{5}$ and illuminated by a paraxial Gaussian beam of radius $w$, namely:

$$
\cos \alpha \exp \left[-2\left(\frac{f \sin \alpha}{w}\right)^{2}\right],
$$

where $f$ is the focal length. While the forms of (6) and (7) are different, there is close resemblance: for small angles $2(1-\cos \alpha) \approx \sin ^{2} \alpha$, hence the two irradiances become approximately equal if we set $2 f^{2} / k w^{2}$ equal to $d=k w_{0}^{2} / 2$, thus establishing direct correspondence between the beam parameters prior to and after focusing. Although the agreement is very close even for small values of $k d$ - see Fig. 4 - we must nevertheless conclude that the scalar field (3) does not represent exactly the field of a Gaussian beam focused by a lens. Rather, it can be thought of as the complete field of a Gaussian wave which is spherical in the far field and is further characterised by boundless angular extent on the surface of a sphere, just as a paraxial Gaussian beam has infinite extent in any transverse plane.

Finally, the wave (1) is merely the lowest order solution from a set containing products of spherical harmonics and spherical Hankel functions of the first kind. ${ }^{1}$ This set can be obtained by transforming (separable) spherical harmonic waves from prolate to oblate spheroidal coordinates using a transformation proposed by Flammer. ${ }^{1,2}$ Similarly, the wave (3) is the lowest order in a new, singularity-free set constructed using spherical Bessel functions of the first kind $j_{n}$, as follows:

$$
\psi_{m n}=\exp (-k d) j_{n}(p) P_{n}^{m}(s) \exp ( \pm i m \varphi)
$$

where $p=k d(\xi-i \eta), P_{n}^{m}(s)$ are associated Legendre functions and $s=(\xi \eta-1) /(\xi-i \eta)$. As we have seen, the distance from the complex point source-sink can be expressed in terms of the oblate coordinates as $R=d(\xi-i \eta)$, hence $p=k R$; likewise $s=(z-i d) / R$. By substituting these identities into expression (8) we obtain a more convenient form

$$
\psi_{m n}=\exp (-k d) j_{n}(k R) P_{n}^{m}\left(\frac{z-i d}{R}\right) \exp ( \pm i m \varphi)
$$


which can be interpreted as a set of spherical harmonic waves centred on a complex point source-sink. The set (9) is identical to the one hypothesised by Couture and Belanger, ${ }^{6}$ except for the use of Bessel instead of Hankel functions. The spherical Bessel functions are singularity free at the origin, unlike the Hankel functions, and are therefore appropriate for describing physically realisable beams. It remains to be determined which types of higher order beams can be generalised in a natural way by the set (9). However, preliminary studies indicate that Laguerre-Gaussian beams are likely candidates.

\section{References}

1. T. B. Landesman and H. H. Barrett, J. Opt. Soc. Am. A 5, 1610 (1988).

2. C. Flammer, Spheroidal Wave Functions (Stanford Univ. Press, Stanford, 1957).

3. C. J. R. Sheppard and S. Saghafi, Phys. Rev. A 57, 2971 (1998).

4. G. A. Deschamps, Electr. Lett. 7, 684 (1971).

5. B. Richards and E. Wolf, Proc. Roy. Soc. London A 253, 358 (1959).

6. M. Couture and P. A. Belanger, Phys. Rev. A 24, 355 (1981). 


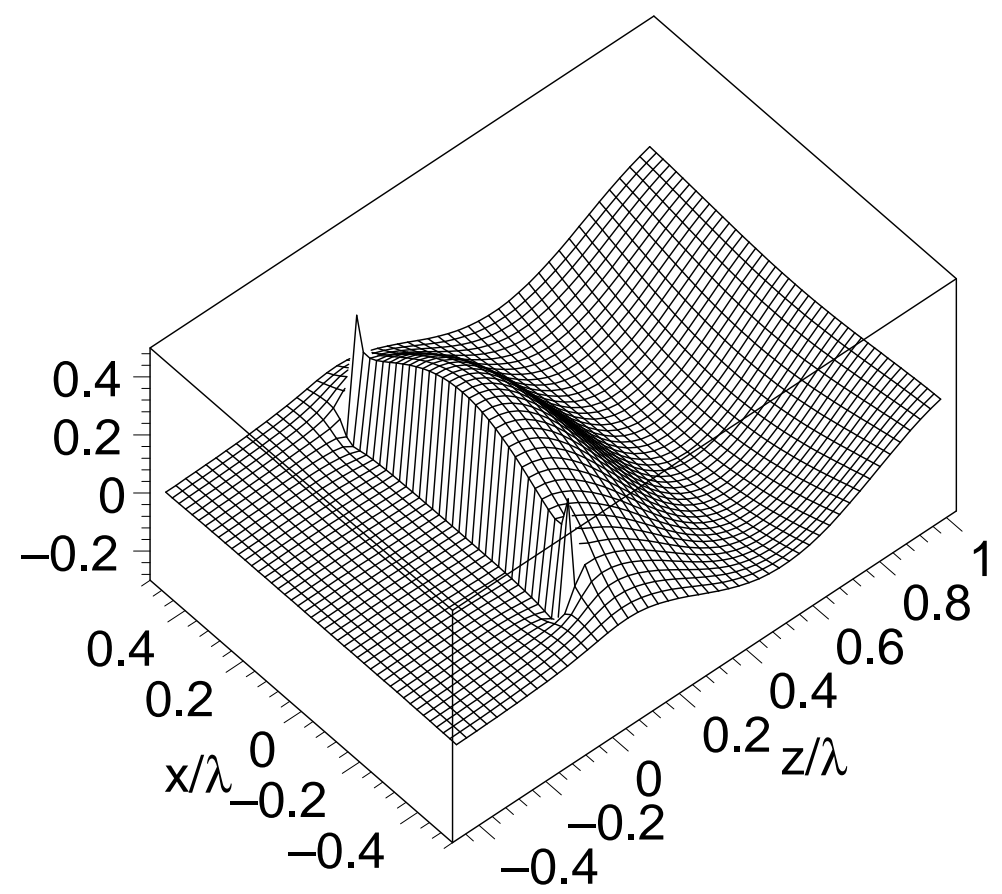

Fig. 1. The real part of the wave (1) plotted in the vicinity of the geometric focus with $k d=2.3$, showing the singularity and the discontinuity.

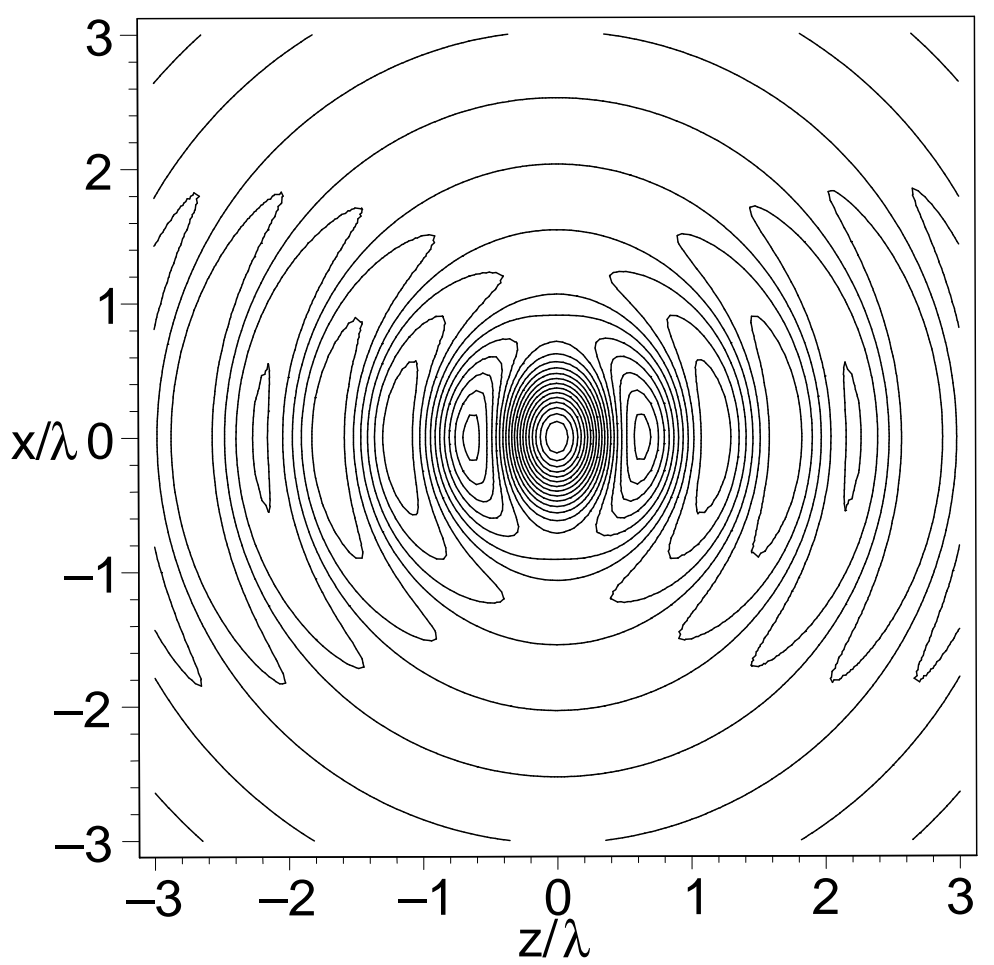

Fig. 2. Field contours at $\mathrm{t}=0$ for the combined wave (3) with $k d=2.3$, shown in the plane of the beam axis. 


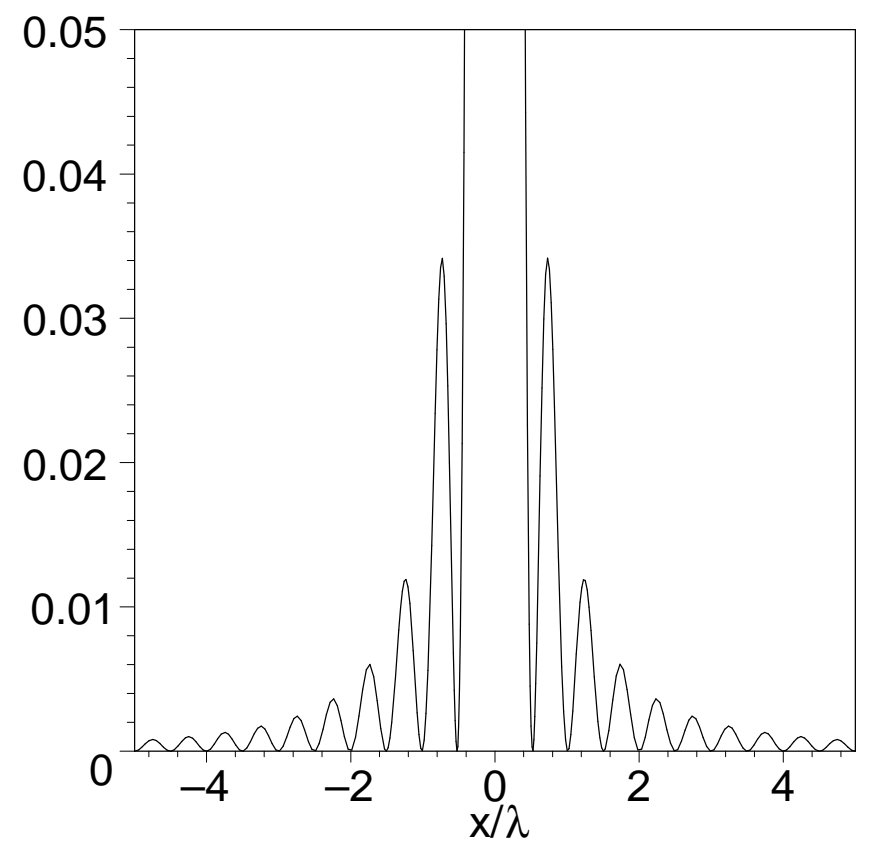

Fig. 3. Time-averaged energy density of the wave (3) with $k d=1$, shown in the focal plane $z=0$ and normalized to 1 at $x=0$.

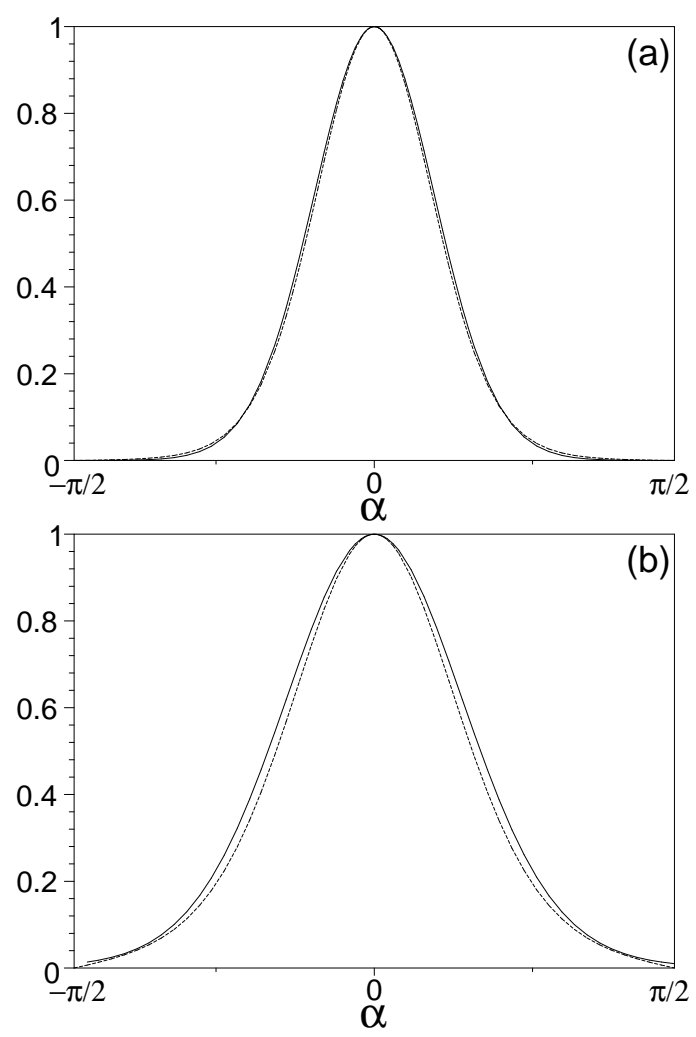

Fig. 4. Far-field irradiance of the Gaussian wave (6) for beams with $k d=5$ (a) and $k d=2.3$ (b) (continuous lines) compared to irradiance at a sine-condition lens (7) (broken lines), plotted against polar angle. The divergence half-angles are $39^{\circ}$ and $69^{\circ}$, respectively. 